\title{
Symbiotic Cognitive Networks: A proposal
}

\author{
Tinku Rasheed ${ }^{1}$ Emma Hart ${ }^{2}$ Jim Bown $^{3}$, Ruth Falconer ${ }^{3}$ \\ Create-Net ${ }^{1}$ Edinburgh Napier University ${ }^{2}$ University of Abertay ${ }^{3}$ \\ tinku.rasheed@create-net. org $^{1}$ e.hart@napier.ac. $\mathrm{uk}^{2}$ \\ j.bown,r.falconer@abertay.ac.uk ${ }^{3}$
}

\begin{abstract}
We describe the concept of a cognitive network and propose that ecosystems of co-existing networks which are globally energy efficient while providing the expected quality of service can be realised by exploiting two mechanisms which occur in biological systems; symbiosis and co-existence.
\end{abstract}

\section{Cognitive Radio Networks}

Cognitive radio is a paradigm for wireless communications in which either a network or a device can change its transmission or reception parameters (power, frequency etc.) to communicate efficiently avoiding interference with adjacent networks. This alteration of parameters is based on the active monitoring of several factors in the external and internal radio environment, such as radio frequency spectrum, user behaviour and network status. Cognitive networks are characterized by flexible network architectures exploiting the capabilities of cognitive radios 2] and have the potential to drive significant innovations [1].

As the global telecommunication industry is gearing itself towards the implementation of wireless networks which are autonomously managed, we can foresee a plethora of flexible and heterogeneous wireless networks including: wide-area terrestrial infrastructure networks, sensor networks, femto-cellular networks, mesh networks, self-organizing ad hoc networks which will need to coexist and cooperate in a global environment. Figure 1 depicts a typical scenario; a single telecom operator leases spectrum in a certain range - within this spectrum, a number of individual (and heterogenous) networks $(\mathrm{CN} 1, \mathrm{CN} 2, \ldots)$ are required to operate without causing interference to each other. In order to minimize interference, each network has two choices:

1. Operate at a unique frequency (depicted by the vertical bars on the right hand graph)

2. Operate at low power which limits interference with other networks (depicted by the horizontal bar).

Therefore, for the global system to function, each of the networks must cooperate and/or collaborate by sharing and/or adapting its resources. Cognitive radio offers the potential to endow the networks with the ability to adapt such that a scalable system of networks can be created, optimised in terms of energy 
efficiency and resource conservation. However, its use to date has been mainly limited to frequency agility, i.e in selecting the best radio access technologies and frequencies at which to operate. In order to fully exploit its potential, we propose to exploit the adaptive capabilities of cognitive radio to create systems of networks. A major challenge is to quantify the degree of interaction between the heterogeneous sub-networks and to enable these sub-networks to operate efficiently without compromising their own resources. Moreover, a unified approach to the design of cognitive networks is necessary to characterize the scale at which the cognitive network interactions can grow and flourish, in a global system of networks.

While designing systems of cooperating networks may be challenging for engineers, it is a problem which has been neatly solved in nature by evolution, which enables stable eco-systems to exist in which multiple species either co-exist - that is, live in separate niches which do not overlap, or exist in symbiotic relationships, a term which was first coined in 1879 to describe 'the living together of unlike organisms'. We propose that co-existence within natural ecosystems has an obvious anaology to property (1) listed above where as symbiotic relationships map neatly to property (2). In the remainder of the paper we outline the main principles of these biological relationships and describe a mapping between the concept of an ecosystem of cognitive radios and biological systems. We propose that by looking in detail at two biological systems - the immune system and fungal communities - we can develop novel techniques for implementing the cognitive network paradigm.

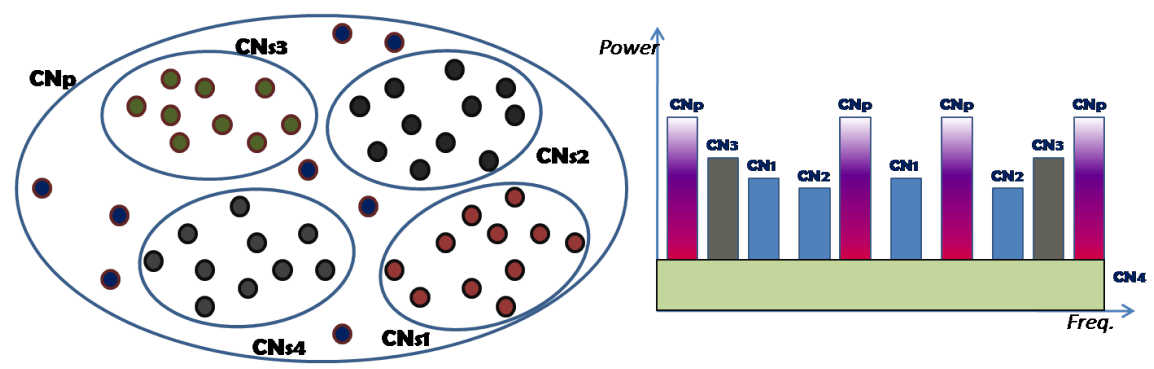

Fig. 1. A system of networks composed of heterogeneous physical sub-networks

\section{Relationships in natural ecosystems}

Symbiotic relationships in nature occur in three forms; commensal, mutualistic and parasitic. The first two are of relevance to our argument. In commensal symbiotic relationships one organism reaps the benefit of another, while the other is not significantly harmed. The mammalian immune system provides an 
excellent example of such a relationship in which some species of bacteria successfully colonize our body and provide us with essential function without which we could not survive, such as stimulation of the gut, immune system development, rejection of pathogenic bacteria, and contributions to our nutrient supply. In mutualistic relationships, both species involved in the relationship benefit. Such relationships are observed between fungi and plants; fungi invade or live amongst the secondary roots of the plant - this benefits the plant which achieves a more efficient mineral uptake and also the fungi which benefit from the sugars translocated to the root by the plant. This relationship is also interesting from the perpective of the plant which could maintain its own mineral supply, but chooses to leave this to the fungi in order to optimize its resources elsewhere. Co-existence relationships are also observed in fungal communities; for examples diferent colonies can co-exist by sharing resource yet maintaing exclusive areas. We propose that by modelling and abstracting these biological processes, it may be possible to construct cognitive networks which can collectively exist within an eco-system that is globally optimal in terms of efficiency and usage of resource. A mapping between the two paradigms is shown in table 1 .

\begin{tabular}{||c|c|c||}
\hline Cognitive Network & Example & Biological System \\
\hline Device & laptop, phone & Organism \\
Network & wireless network & Species \\
Network of Networks & spectrum licenced by single operator & ecosystem \\
\hline
\end{tabular}

Table 1. Mapping between biological and cognitive networks

\section{Future Work}

We propose to investigate the possibility of creating scalable, adaptive ecosystems of networks by looking to biology. In summary, the utimate goals of our work are to explore theoretical models and abstract mechanisms of symbiosis to identify and model the interaction patterns of symbiotic relationships in light of the requirements stemming from future generation wireless networks; to quantify the amount of resources required for specific operations in coexisting networks, thereby conceiving an overall eco-footprint model for cognitive networks via adapted metrics derived from the proposed mapping; and to realize a unified framework for symbiotic cognitive networking synthesized from a novel multidisciplinary scientific approach.

\section{References}

1. Cognitive radio. MIT Technology Review, March-April.

2. C. Fortuna and M. Mohorcic. Trends in the development of communication networks: Cognitive networks. Computer Networks, 2009. 